\title{
Diagnosis, Management and Outcomes of Intestinal Obstruction at an Urban Tertiary Hospital in Sub Saharan Africa: a Cross-Sectional Study.
}

\section{Arlene Muzira Nakanwagi}

Makerere University

Stephen C Kijjambu

Makerere University

Tonny Stone Luggya ( $\sim$ tluggya@chs.mak.ac.ug )

Makerere University

\section{Research Article}

Keywords: Acute Abdomen, Intestinal Obstruction,

Posted Date: March 10th, 2021

DOl: https://doi.org/10.21203/rs.3.rs-264407/v1

License: (1) This work is licensed under a Creative Commons Attribution 4.0 International License.

Read Full License

Version of Record: A version of this preprint was published at BMC Surgery on November 30th, 2021. See the published version at https://doi.org/10.1186/s12893-021-01405-x. 


\section{Abstract}

BACKGROUND: Intestinal Obstruction (IO) is among the commonest causes of acute abdomen worldwide and globally it remains a challenge because it is a major cause of morbidity and surgical financial expenditure. Clinically it presents with nausea, vomiting, colicky abdominal pain and cessation of bowel movements or passage flatus and stool. Diagnosis can be clinical but is confirmed usually by radiologic imaging. We studied the current diagnosis, management and outcomes of 10 in Mulago Hospital

MATERIALS AND METHODS: This was a Prospective Descriptive Study in all the surgical units of Mulago from January to May 2014. Ethical approval was got in line with Helsinki declaration and then a pretested and validated questionnaire was used to collect data. Informed consent was got with eligible and consenting/assenting patients recruited among those patients of all ages and sex presenting with suspected Intestinal Obstruction. Uni-variate and bi-variate of the variables plus measurements of associations were done.

RESULTS: We enrolled 135 patients, excluded 25 and recruited 110 patient with more males than females i.e. $71.8 \%$ males and $28.2 \%$ females. Colicky abdominal pain, abdominal distension, and vomiting were the 3 commonest symptoms with abdominal distension, increased bowel sounds and abdominal tenderness as commonest signs. Majority of the patients were diagnosed radiologically (51\%) and the rest (48.2\%) clinically diagnosed accounting. "Dilated bowel loops" was the most frequent radiological sign. Return of bowel sounds occurred within 5 days of the POD, while opening of bowels on average, occurred on the $3^{\text {rd }}$ POD. The mean day of discharge was the $5^{\text {th }}$ POD and $73 \%$ of the patients were discharged by the $7^{\text {th }}$ Post-Operative Day. The commonest unfavourable management outcome noted was prolonged hospital stay followed by wound sepsis (Surgical Site Infection) and Mortality

CONCLUSION: Majority of the patients were diagnosed radiologically (51\%). surgical management was done for $72.7 \%$ of the cases and $27.3 \%$ conservatively managed. Prolonged hospital stay was the commonest unfavorable outcome of management

\section{Background}

Intestinal Obstruction (IO) refers to the interruption of forward flow of intestinal contents and can occur at any point from mouth to anal canal with clinical symptoms varying based on the level of obstruction [1]. It is among the commonest causes of acute abdomen worldwide and is characterized by abdominal pain which in 2006 was the most common reason for a visit to the emergency department in the USA accounting for 8 million (7\%) of the 119 million visits [2]. 10 is a common surgical emergency that is frequently encountered in abdominal surgery $[3,4]$ and it remains a challenge because it is a major cause of morbidity plus financial expenditure worldwide [5]. Clinical presentation of IO generally includes nausea, vomiting, colicky abdominal pain and cessation of bowel movements or passage flatus and stool, although the severity of these clinical symptoms varies based on the acuity and anatomic level of obstruction $[1,6]$. Diagnosis is confirmed usually by radiologic imaging although, with negative 
radiography and high index of suspicion, non-contrast Computed Tomography (CT) scan is recommended [1]. Management of uncomplicated obstructions can be conservative by fluid resuscitation to correct the metabolic derangements, intestinal decompression, and bowel rest. If there's evidence of vascular compromise or perforation then management is by surgical intervention [6].

Recent studies on 10 in Uganda have only been documented in 2 non-governmental hospitals in 2 regions of Uganda [7, 8], yet the tertiary health care institution Mulago National Referral and Teaching Hospital (MNRTH) which offers free services to patients doesn't have recent studies with the last documented IO study done by Macadam in 1961[9]. Also raw data from the Accident and Emergency (A\&E) revels that an average of 50 patients are admitted per month with a suspected diagnosis of IO [10].

We studied the current diagnosis, management and outcomes of IO in MNRTH due to the paucity of data to guide current policy on decision making process, resuscitation measures, timing of surgery and choice of surgical procedure in our setting.

\section{Materials And Methods}

\section{Study Setting}

MNRTH, located in Kampala, the Capital City of Uganda, is a 1500 bed tertiary hospital caring for approximately 140,000 patients annually, with an annual average of 48,000 patients transitioning through the A\&E[11]. It has three surgical wards, with an A\&E that triages patients into elective or emergency cases done in casualty theatre that records about 31 operations monthly for the relief of IO [10]. Also A\&E records show that on average about 7 to 10 patients' with $I 0$ are seen per week by the Senior House Officer's (SHO's).

\section{Ethical Approval and recruitment}

Ethical approval was obtained from the Department of Surgery, Makerere University School of Medicine Research an Ethics Committee (SOMREC) and MNRTH IRB to conduct a Prospective Descriptive Study in all the surgical units of MNRTH.

It was a prospective descriptive cross sectional study of 110 patients selected by the convenient sampling method. The study population consisted of both male and female patients of all ages, who presented to the surgical units with, suspected IO.

Consent was got from all patients and for subjects below 18 years, a parent, legal guardian or Next Of Kin gave consent on their behalf.

Eligible and consenting patients were recruited with details of their demographics, symptoms and signs recorded.

Aspects of their resuscitation, investigations and treatment were recorded and monitored till discharge. The causes of IO, and the early management outcomes for the first 7 post-operative/post admission days, including deaths or up to discharge, whichever came first, were recorded. 


\section{Study Periods}

The study was conducted from January 2014 and May 2014 where we recruited consented patients that met the inclusion criteria.

\section{Study variables}

The main variables of interest were clinical examination, radiologic findings, and management options focusing on favorable and unfavorable outcomes in the Post-Operative Day (POD) window.

\section{Data management and analysis}

A structured pretested and validated questionnaire was used to collect data. The data was cleaned, backed up and later analyzed in STATA version10.1. Study statistics were reported using proportions, means, medians and inter-quartile ranges. Uni-variate and bi-variate analysis of the variables plus measurements of associations were done using Pearson $C h i-X^{2}$ values, $P$ values and logistic regression. Presentation of data was by bar graphs, pie charts, Box-plots and Tables.

\section{Results}

We recruited 110 patients that met criteria or had outcomes of interest i.e. investigations suggestive of obstruction, or confirmation of diagnosis at laparotomy with favorable and unfavorable outcomes. Demographically there were more males than females i.e. $71.8 \%$ males and $28.2 \%$ females in a ratio of 2.6:1. The 3 commonest symptoms reported by the participants were; colicky abdominal pain, abdominal distension, and vomiting. The 3 commonest examination signs were; abdominal distension, increased bowel sounds and abdominal tenderness. Majority of the patients were diagnosed radiologically (51\%) with clinical diagnosis accounting for $48.2 \%$. However for the 80 cases eventually operated a higher majority (58.8\%) were diagnosed clinically. See Table1

Table 1

\section{Mode of Diagnosis by choice of management}

\begin{tabular}{|lllll|}
\hline characteristic & $\begin{array}{l}\text { Overall } \\
\text { 110(100\%) }\end{array}$ & $\begin{array}{l}\text { Non-operative } \\
\mathbf{3 0 ( 2 7 . 3 \% )}\end{array}$ & $\begin{array}{l}\text { Operative } \\
\mathbf{8 0}(72.7 \%)\end{array}$ & $\begin{array}{l}\text { p-value } \\
\text { (non-operative vs. operative) }\end{array}$ \\
\hline Diagnosis $\mathrm{n}(\%)$ & $57(51.8)$ & $24(80.0)$ & $34(41.3)$ & $0.001^{*}$ \\
Radiological & $53(48.2)$ & $06(20.0)$ & $46(58.8)$ & \\
Clinical & & & & \\
\hline
\end{tabular}

"Dilated bowel loops" was the most frequent sign found on both imaging modalities. Multiple air fluid levels were observed in $12.7 \%$ of the radiographs and faecal impaction was noted in $5.5 \%$ of the x-rays. On Sonography, reversed peristalsis was noted in $10 \%$ of the cases. The other signs were seen $5.5 \%$ of 
the cases. Ultra sound Sonography exerted more influence on the choice of management when compared to plain radiography. $(p=0.001$ vs. $p=0.013)$. Shown in Table 2

Table 2

Radiological signs by choice of management

\begin{tabular}{|lllll|}
\hline Radiological signs & Overall & Non-operative & Operative & p value \\
& $\mathbf{3 3 ( 3 0 \% )}$ & $\mathbf{3 0 ( 2 7 . 3 )}$ & $\mathbf{8 0}(72.7)$ & \\
\hline Plain x-ray & $32(29.1)$ & $14(46.7)$ & $18(22.5)$ & 0.013 \\
Dilated bowel loops & $29(26.4)$ & $12(40.0)$ & $17(21.3)$ & $<0.001$ \\
Multiple air levels & $14(12.7)$ & $01(3.3)$ & $13(16.3)$ & 0.07 \\
Faecal impaction & $06(5.5)$ & $06(20.0)$ & $00(0.0)$ & 0.047 \\
\cline { 1 - 2 } Sonographic findings & $33(30.0)$ & $16(53.3)$ & $17(21.3)$ & 0.001 \\
Dilated bowel loops & $29(26.4)$ & $12(40.0)$ & $17(21.3)$ & 0.047 \\
Reversed/no peristalsis & $11(10.0)$ & $05(16.7)$ & $06(07.5)$ & 0.154 \\
Doughnut sign & $06(05.5)$ & $02(06.7)$ & $04(05.0)$ & 0.732 \\
Abdominal masses & $06(05.5)$ & $05(16.7)$ & $01(01.3)$ & 0.002 \\
Peritonitis & $06(05.5)$ & $00(00.0)$ & $06(05.5)$ & 0.161 \\
\hline
\end{tabular}

Outcomes were categorized as favourable or unfavorable with the favourable outcomes being: return of bowel sounds, opening of bowel, NG-tube removal, drain removal and discharge by the 7th post-operative or post admission day (for non-operative management). The unfavorable outcomes included: wound sepsis, systemic sepsis, anastomotic leak, anemia, chest infections, prolonged hospital stay and death.

For the favourable outcomes; most post-operative events occurred within the first 10 days. Return of bowel sounds occurred within 5 days of the POD, while opening of bowels on average, occurred on the 3rd POD. The mean day of discharge was the 5th POD and 73\% of the patients were discharged by the 7th Post-Operative Day (Fig. 1).

The commonest unfavourable management outcome noted was prolonged hospital stay followed by wound sepsis (Surgical Site Infection) and Mortality (Fig. 2).

Factors that were persistently associated with favorable outcomes, in the Uni-variate model, were fever $(\mathrm{RR}=0.19, p=0.09)$, Increased bowel sounds $(\mathrm{RR}=4.3, p=0.001)$. Absent bowel sounds and abdominal tenderness $(p=0.001 \& p=0.014)$ showed association with favorable outcomes at lower odds. Small Bowel Obstruction (SBO) is 3.17 likely to be associated with favorable outcomes $(p=0.013)$. See Table 3 . 
Table 3

Displays modified Cox regression analysis used to assess the significant factors associated with outcome $(p<0.05)$. These were selected for this further analysis.

\begin{tabular}{|c|c|c|c|}
\hline Predictor of outcome & Relative- Risk & Confidence interval & P-Value \\
\hline Symptom duration & 1.83 & $0.289-12.07$ & 0.528 \\
\hline 24-48 hours & 0.67 & $0.149-2.970$ & 0.596 \\
\hline 48-72 hours & 0.52 & $0.132-2.012$ & 0.340 \\
\hline$>72 \mathrm{hrs}$ at home & 0.29 & $0.075-1.112$ & 0.075 \\
\hline \multicolumn{4}{|l|}{$>72 \mathrm{hrs}$ in health center } \\
\hline Operative Vs. Non-operative management & 0.21 & $0.067-0.650$ & 0.007 \\
\hline Symptoms of 10 & 0.93 & $0.382-2.256$ & 0.870 \\
\hline Abdominal pain & 0.19 & $0.540-0.665$ & 0.009 \\
\hline Fever & 0.59 & $0.220-1.181$ & 0.116 \\
\hline Vomiting & 0.67 & $0.297-1.533$ & 0.348 \\
\hline Relative constipation & 1.33 & $0.537-3.280$ & 0.540 \\
\hline \multicolumn{4}{|l|}{ Absolute constipation } \\
\hline Signs & 0.56 & $0.202-1.563$ & 0.270 \\
\hline Abdominal distension & 0.24 & $0.968-0.522$ & 0.001 \\
\hline Absent bowel sounds & 4.28 & $1.855-9.861$ & 0.001 \\
\hline Increased bowel sounds & 0.36 & $0.162-0.817$ & 0.014 \\
\hline Abdominal tenderness & 1.61 & $0.162-16.02$ & 0.685 \\
\hline \multicolumn{4}{|l|}{ Percussion tenderness } \\
\hline Sonographic signs of IO & 0.398 & $0.113-1.402$ & 0.152 \\
\hline Reversed peristalsis & 0.333 & $0.053-2.088$ & 0.241 \\
\hline Peritonitis & 1.058 & $0.185-6.061$ & 0.969 \\
\hline \multicolumn{4}{|l|}{ Abdominal masses } \\
\hline Clinical Vs. Radiological diagnosis & 0.762 & $0.346-1.674$ & 0498 \\
\hline Level of obstruction(SBO vs. LBO) & 3.17 & $1.280-7.861$ & 0.013 \\
\hline
\end{tabular}




\begin{tabular}{|llll|}
\hline Predictor of outcome & Relative- Risk & Confidence interval & P-Value \\
\hline Bowel status & 0.50 & $0.155-1.611$ & 0.245 \\
Strangulation & 0.20 & $0.059-0.723$ & 0.014 \\
Gangrene & 0.44 & $0.090-2.189$ & 0.319 \\
Perforation & 0.25 & $0.508-1.231$ & 0.088 \\
peritonitis & & & \\
\hline Urethral catheterization & 0.22 & $0.617-0.789$ & 0.020 \\
\hline
\end{tabular}

The choice of management, i.e. non-operative versus operative, greatly influenced outcome with more of the patients undergoing non-operative management experiencing favourable outcomes.

The results in Table 4 below show the recomputed odds ratio in a multivariate regression and unlike in the binary logistic regression, none of the factors independently predict the outcome, implying that they most likely act in association or in combination with one another, as their Relative Risk changes and the $\mathrm{P}$ value becomes statistically insignificant.

Table 4

Multi-variate analysis of statistically significant variables in the Uni-variate regression

\begin{tabular}{|llll|}
\hline Predictor of outcome & Relative-Risk & Confidence interval & $P$ value \\
\hline Choice of management(operative) & 0.39 & $0.111-1.342$ & 0.134 \\
\hline Level of obstruction & 1.85 & $0.622-5.592$ & 0.266 \\
\hline Signs of IO & 0.28 & $0.599-1.335$ & 0.111 \\
Fever & 1.35 & $0.176-10.34$ & 0.770 \\
Increased bowel sounds & 0.48 & $0.058-3.946$ & 0.493 \\
Absent bowel sounds & 0.72 & $0.255-2.035$ & 0.530 \\
Abdominal tenderness & 6.99 & $0.545-89.81$ & 0.140 \\
Percussion tenderness & & & \\
\hline
\end{tabular}

\section{Discussion}

We studied the diagnosis, management and early outcomes 10 in our local setting at MNRTH. Baseline demographics showed more males than females i.e. $71.8 \%$ males and $28.2 \%$ females. Radiological imaging was done for $51.8 \%$ while $48.2 \%$ were diagnosed clinically, this significant role of 
clinical diagnosis is comparable to that in other African studies [12, 13, 14]. The modality of diagnosis influenced choice of management and the diagnostic value of radiographs was depicted in the strong statistical correlation between imaging and choice of management (Table 2), with X-rays having a $p=$ 0.013 , while Sonography had a $p=0.001$. Studies have shown that there is also increasing reliance on radiological investigations when the immediate choice of management is conservative rather than operative because it's shown that a proportion of cases will resolve spontaneously [15].

We noted that Eighty $(72.7 \%)$ of the participants who presented with 10 were managed operatively with resection and anastomosis as the commonest procedure done on 28 (35.0\%) followed by hernia repair at $23.8 \%$ and then Simple colostomy at $15 \%$ as the 3rd commonest procedure and laparotomy with colostomy was the least common procedure in the study. Seventy three (66.4\%) cases had favourable outcomes while $37(33.6 \%)$ had associated morbidity and mortality. Prolonged hospital stay (30.4\%) which was the commonest adverse outcome was comparable to regional studies that had similar findings $[7,8,16,17,18]$.

The procedure most associated with prolonged hospital stay was resection and anastomosis, followed by ileostomy and we posit that this was so because cases diagnosed as "tumour" had to undergo radiotherapy treatment or staging investigations prior to definitive management. We also found out that mortality was the 3rd commonest postoperative complication at a frequency of $8.8 \%$ which was comparatively low compared to similar studies that showed rates of $12.9 \%, 19.7 \%$ and $20 \%[8,17,19,20]$.

Our rates of non-operative management in this study (27.3\%) compare differently to those in developed countries like USA (73\%) and we postulate that could probably be attributed to the lack of alternative methods of non-operative management such as Gastrograffin use in adhesions, hydrostatic or pneumatic reduction of intussusception, as well as the lack of investigative capacity to confirm or rule out bowel ischemia in our setting $[16,21,22]$.

This study was subject to limitations such as recall bias as some patient data was from records and POD follow up interviews of the operating surgeon or $\mathrm{SHO}$, this may have affected some study variables collected.

\section{Conclusion}

In conclusion, majority of the patients were diagnosed radiologically ( $51 \%)$ with clinical diagnosis accounting for $48.2 \%$ however both remain complimentary 10 diagnostic factors. $72.7 \%$ of the cases were surgically managed with $27.3 \%$ conservatively managed. Prolonged hospital stay was the commonest unfavorable outcome of management followed by wound sepsis and then death.

\section{List Of Abbreviations}

MNRTH: Mulago National Referral and Teaching Hospital 
IO: Intestinal Obstruction

SBO: $\quad$ Small Bowel Obstruction

LBO: $\quad$ Large Bowel Obstruction

POD: $\quad$ Post-Operative Day

PAD: $\quad$ Post Admission Day

SHO's: $\quad$ Senior House Officers

\section{Declarations}

ETHICS APPROVAL AND CONSENT: We obtained ethical approval from Department of Surgery, Makerere University's SOMREC and MNRTH Research and Ethics Board. Informed consent was obtained from all adult study subjects and for those below 18 , informed consent (assent) was by the parents, legal guardian or Next of Kin.

CONSENT FOR PUBLICATION: The authors give consent for publication

AVAILABILITY OF DATA AND MATERIALS: The data supporting the findings of this study, other than that is available within the article, are available upon request from the corresponding author

COMPETING INTERESTS: The authors declare that they have no conflict of or competing interests either financial or non-financial.

FUNDING: This was self-funded academic research for Masters in Medicine degree.

DUPLICATE PUBLICATION: This is an original manuscript whose work is not under consideration elsewhere, copyright has not been breached in seeking publication and the publication has been approved by all co-authors and responsible authorities at MNRTH

\section{AUTHOR CONTRIBUTION:}

- Dr. AMN: developed this idea from conception, proposal development, data collection and manuscript writing as this was her thesis for her masters in Surgery.

- Dr. SCK and PO(RIP) gave surgical supervisory oversight that helped oversee the proposal development and final proposal preparation

- Dr. TSL worked with Dr. AMN worked hand in hand to develop this into a final proposal through to data collection and manuscript writing for this submission.

\section{ACKNOWLEDGEMENT:}


- To all the colleagues in the Department of Surgery at Makerere University whose guidance and instruction was invaluable especially my supervisors for their unwavering commitment.

- To all the SHO's graduate colleagues with unique personalities, individual strengths and "fine hands who walked the data collection journey and made it lighter".

- The tireless and dedicated staff of Mulago National Referral and Teaching Hospital who despite the odds stacked against them keep on keeping on with working of saving lives

\section{References}

1. Jackson PG, Raiji MT. Evaluation and management of intestinal obstruction. Am Fam Physician. 2011 Jan 15;83(2):159-65. PMID: 21243991.

2. Pitts SR, Niska RW, Xu J, Burt CW. National Hospital Ambulatory Medical Care Survey: 2006 emergency department summary. Natl Health Stat Report. 2008 Aug 6;(7):1-38. PMID: 18958996.

3. Mucha P Jr. Small intestinal obstruction. Surg Clin North Am. 1987 Jun;67(3):597-620. doi: 10.1016/s0039-6109(16)44234-9. PMID: 3296252.

4. Miller G, Boman J, Shrier I, Gordon PH. Natural history of patients with adhesive small bowel obstruction. Br J Surg. 2000;87:1240-1247

5. Miller G, Boman J, Shrier I, Gordon PH. Etiology of small bowel obstruction. Am J Surg. 2000;180:33-36.

6. Jackson P, Vigiola Cruz M. Intestinal Obstruction: Evaluation and Management. Am Fam Physician. 2018 Sep 15;98(6):362-367. PMID: 30215917.

7. Kakande I, Ekwaro I, Obote W, Nassali G, Kyamanywa P. The intestinal Volvulus at St. Francis Hospital, Kampala. East and Central Afr J Surg. 2003;6(1):21-4.

8. Okeny P, Hwang T, Ogwang D. Acute Bowel Obstruction in a Rural Hospital in Northern in Northern Uganda. East and Central African Journal of Surgery. 2011;16(1).

9. McAdam I. A three year review of intestinal obstruction: Mulago Hospital, Kampala, Uganda. East African medical Journal. 1961;38:536-43.

10. Mulago- Hospital Records. Jan 2007-dec 2008. Accident and emergency theatre operations registry. (Un published data)

11. Tran TM, Fuller AT, Kiryabwire J, Mukasa J, Muhumuza M, Ssenyojo H, Haglund MM. Distribution and characteristics of severe traumatic brain injury at Mulago National Referral Hospital in Uganda. World Neurosurg. 2015 Mar;83(3):269-77. doi: 10.1016/j.wneu.2014.12.028. Epub 2014 Dec 19. PubMed PMID: 25529531.

12. Cole G. A review of 436 cases of intestinal obstruction in Ibadan. Gut. 1965;6(2):151-62.

13. Kitara DL, Kakande I, Mugisa B. The Possum scoring systems for patients undergoing Laparotomy at Mulago Hospital. East Central African Journal of Surgery. 2007;12(2):133-42.

14. Shittu O, Gana J, Alawale E, Ogundiran T. Pattern of mechanical intestinal obstruction in Ibadan: a ten year review. African journal of medicine and medical sciences. 2001;30(1-2):17. 
15. Burkill G, Bell J, Healy J. Small bowel obstruction: the role of computed tomography in its diagnosis and management with reference to other imaging modalities. Eur Radiol. 2001;11(8):1405-22. doi: 10.1007/s003300100882. PMID: 11519550.

16. Kintu-Luwaga R, Galukande M, Owori FN. Serum lactate and phosphate as biomarkers of intestinal ischemia in a Ugandan tertiary hospital: a cross-sectional study. International journal of emergency medicine. 2013;6(1):1-7.

17. Ntakiyiruta G, Mukarugwiro B. The Pattern of intestinal Obstruction at Kibogola Hospital, a Rural Hospital in Rwanda. East and Central African Journal of Surgery. 2009; 14(2):103-8.

18. Madziga A, Nuhu A. Causes and treatment outcome of mechanical bowel obstruction in north eastern Nigeria. West African journal of medicine. 2008;27(2):101

19. Sourkati E, Fahal A, Suliman S, ARABI Y. Intestinal obstruction in Khartoum. East African medical journal. 1996;73(5):316-9.

20. Doumi E, Mohammed M. Acute Intestinal Obstruction in El Obeid Hospital, Western Sudan. Sudan Journal of Medical Sciences. 2008;3(3):191-6.

21. Shih S-C, Jeng K-S, Lin S-C, Kao C-R, Chou S-Y, Wang H-Y, et al. Adhesive small bowel obstruction: How long can patients tolerate conservative treatment? World journal of Gastroenterology. 2003;9(3):603-5.

22. Choi H-K, Chu K-W, Law W-L. Therapeutic value of gastrografin in adhesive small bowel obstruction after unsuccessful conservative treatment: a prospective randomized trial. Annals of surgery. 2002;236(1):1.

\section{Figures}

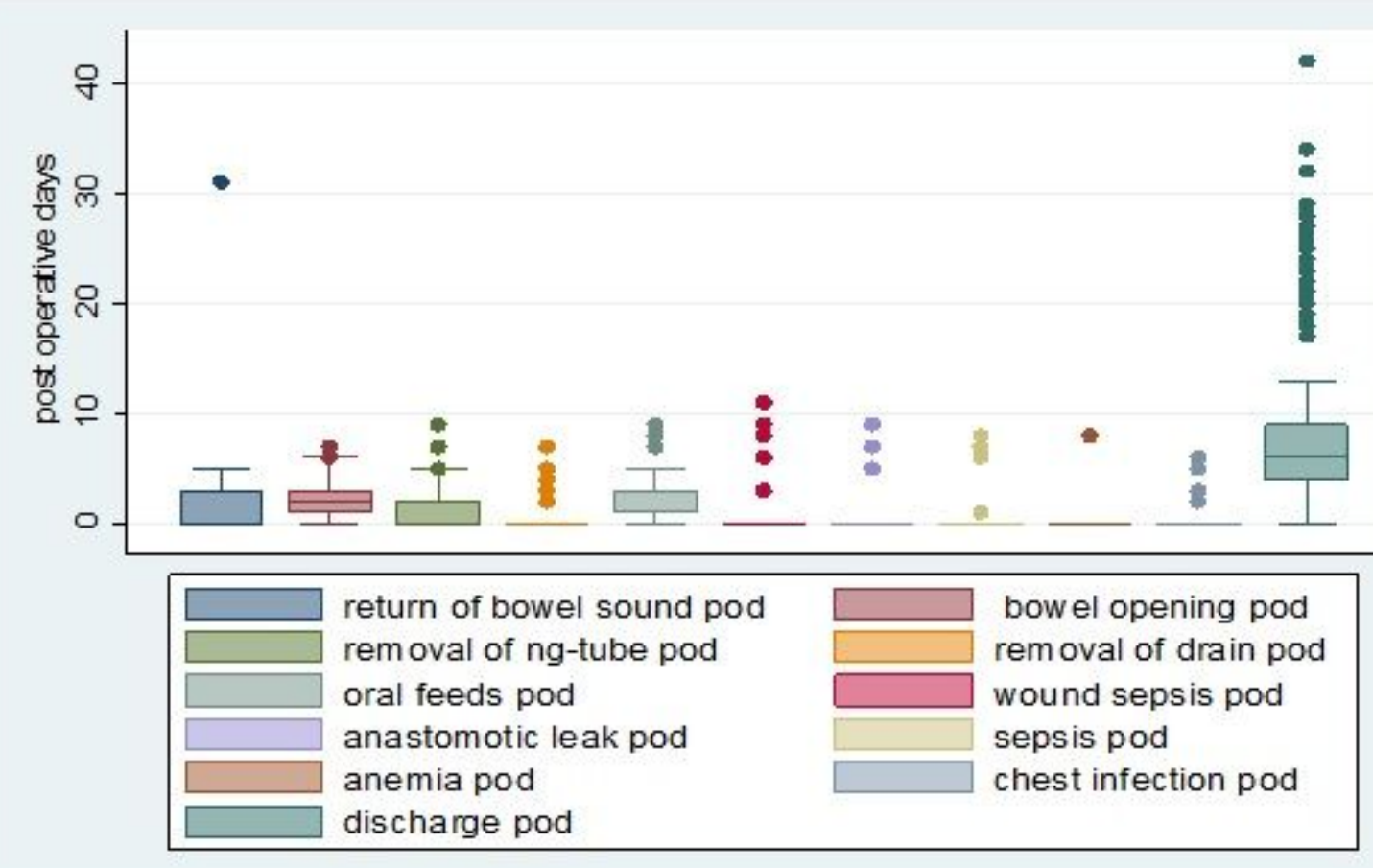


Figure 1

Box plot showing post-management events by Post-operative/ admission day

\section{percentage of cases}

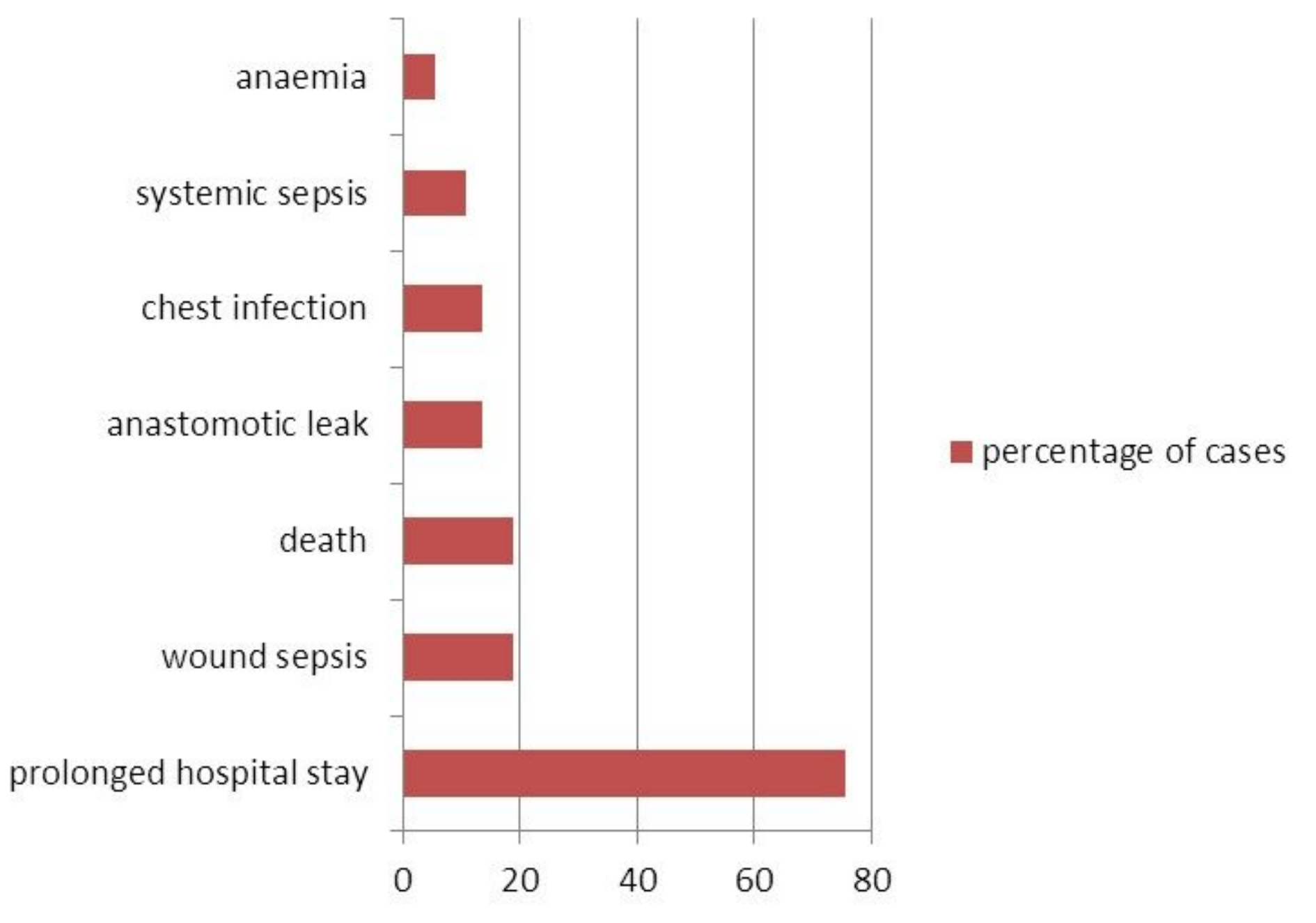

Figure 2

Bar graph showing percentage distribution of unfavourable outcomes. 